\title{
Development of dye sensitized solar cells
}

\author{
Mengdan Chen $^{1}$ \\ ${ }^{1}$ School of Chemistry and Chemical Engineering, Beijing Institute of Technology, Beijing 100081, China
}

\begin{abstract}
With the development of society, the demand for energy is increasing significantly, and the environmental problems are becoming more and more serious. Therefore, it is urgent to find efficient and clean new energy. Among the many new energy sources, solar energy has been favoured most for its universality, harmlessness and low cost. In 1991, the photoelectric conversion efficiency of dye-sensitized solar cells (DSSCs) has been greatly improved, which has attracted the attention. In recent 30 years, the researches on DSSCs have been increasing and expanding. Dye sensitizer is the most important component of DSSC, and also a key issue of researchers. This paper aims to summarize the types, structures and development trends of dye sensitizers, and provide inspiration for us to design and evaluate new dye sensitizers.
\end{abstract}

\section{Introduction}

With the rapid development of society, our lives are increasingly dependent on energy. A large number of nonrenewable energy is constantly exploited and utilized which has caused serious environmental problems. Therefore, access to clean, cheap, reliable and sustainable new energy is the cornerstone of the world's growing prosperity and economic growth in the 21 st century [1]. Then a large number of new renewable energy emerged, such as solar energy, wind energy, geothermal energy, tidal energy and so on [2]. Solar energy has been vigorously studied and developed because of strong adaptability, low cost and little environmental harm [3]. So far, solar cells have been widely used in people's production and life.

Among many kinds of solar cells, dye-sensitized solar cells (DSSCs) have developed rapidly in recent years, and their research history can be traced back to the 1960s. Michael Grätzel and coworkers have been working on dye-sensitized $\mathrm{TiO}_{2}$ thin film solar cells since the 1980s [4-5]. The DSSCs prepared by $\mathrm{TiO}_{2}$ film, efficient ruthenium-pyridine dye, $\mathrm{I}^{-} / \mathrm{I}_{3}^{-}$electrolyte system and platinum modified counter electrode. Dye-sensitized solar cell has been widely and continuously studied to make great progress and becomes one of the mainstream solar cells, since O'Regan and Grätzel announced a high solar energy conversion efficiency of $7.1 \%$ in 1991 [6].

Generally speaking, dye sensitizer is composed of electron donor (D), conjugated bridge ( $\pi$-linker) and electron acceptor (A) according to several frameworks, such as D-A, D- $\pi$-A, D-A- $\pi$-A, etc. In addition, dye sensitizers can be divided into metal complex dye sensitizers and metal-free organic dye sensitizers according to the different composition materials. The cost of metal complex dyes is restricted by the price of rare metals (such as ruthenium). Therefore the research and development of efficient metal-free organic dyes are an effective means to expand production and reduce costs.

The challenge to improve the performance of DSSC is the chemical complexity of the device. There are complex interactions among all components, which determine the final performance of solar cells. Therefore, a lot of repetitive and tedious work is needed to complete the optimization of each component in the experiment. The application of theoretical chemical calculation in the study of DSSCs is of great significance to deeply understand the structure-activity relationship and the main role of each component from the microscopic nature [7]. It can not only optimize the components systematically and efficiently, but also effectively save material and financial resources, reduce the research cost of DSSCs, and help its further development.

\section{Construction and operating principles of DSSCs}

Normally, a typical DSSC system consists of the nanocrystalline semiconductor photoanode, dye sensitizer, redox electrolyte solution, counter electrode and conductive substrate [8]. The complete working principle of DSSC device can be described into the several steps as shown in Fig.1. Firstly, dye sensitizer is converted into excited state from ground state by the irradiation of sunlight. Secondly, the excited electrons transfer into the semiconductor film, and then diffuse through the conductive substrate and enter the external circuit. Thirdly, the oxidized dye is regenerated by the reduction of the reductive electrolyte, while the oxidized electrolyte is reduced by the electrons from counter electrode [9]. Aaccording to the working principle of DSSCs, energy levels of the highest occupied molecular orbital (HOMO) and the lowest unoccupied molecular orbital (LUMO) are 
bounded. That is the level of HOMO under the redox potential of $\mathrm{I}^{-} / \mathrm{I}_{3}{ }^{-}$electrolyte $(-4.8 \mathrm{eV} v s$. vacuum $)$ and the level of LUMO upon the $\mathrm{CB}$ of $\mathrm{TiO}_{2}(-4.0 \mathrm{eV}$ vs. vacuum) which can ensure the effective injection of excited electrons [10]. Therefore, the interactions between semiconductor materials, electrolyte and dye sensitizers have a great influence on the electron transport in DSSC, which is the main determinant of DSSC performance.

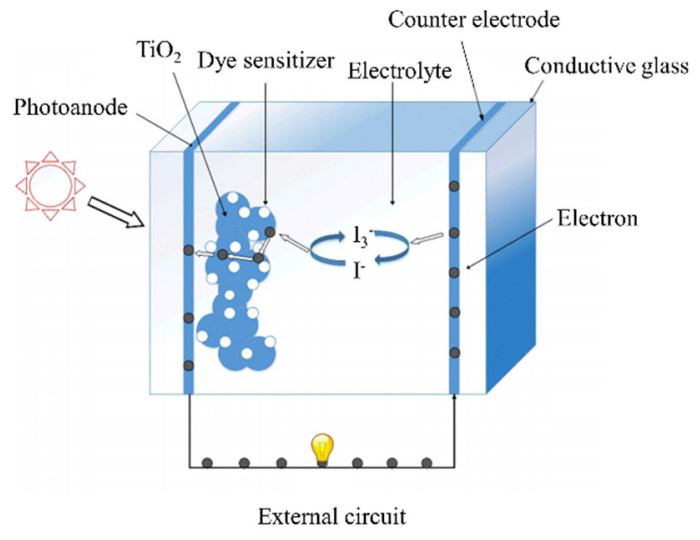

Fig. 1. Scheme of a DSSC.

\section{Classical structures of dye sensitizers}

As an important part of DSSCs, dye sensitizers have been deeply studied by many researchers [11]. By analyzing dye sensitizer, we can conclude that the dye should have a wide range of absorption in the visible light, so as to promote the excitation of electrons [12]. Secondly, the dye should have strong electron donating ability to promote the electron excitation into the conduction band of semiconductor materials. Therefore, dye molecules should have at least the following three parts: electron donor, electron acceptor and $\pi$-linker [13]. Most of the donors are electron rich groups such as triphenylamine and trimethoxybenzene. In addition to transfer electrons, acceptors need to be helpful to combine with semiconductor materials. Most of the $\pi$-linker are planar and strongly conjugated groups, such as heterocyclic rings, thiophene, and furan and so on. In order to achieve better electron excitation and injection efficiency, the structure of dye sensitizers has also undergone a series of changes and there are two classical frameworks D-A and D- $\pi-A$.

\subsection{D-A framework [14]}

This type of dye sensitizer consists of only two parts: donor and acceptor, which is the simplest structure as dye sensitizer [15]. The advantage of this kind of dye sensitizer is they do not contain $\pi$-linkers. On the one hand, that shortens the length of dye molecules so as to quickly transfer electrons from donor to acceptor. On the other hand, most of the $\pi$-linkers have strong conjugation. So when the dyes aggregate on the semiconductor surface, the $\pi$-linkers are easy to interact with each other, which seriously affect the absorption of visible light and results in the loss of energy.

\subsection{D-T-A framework}

$\mathrm{D}-\pi$-A architecture is a promising strategy for dye design, which has excellent photoelectric conversion and electron transfer ability. These advantages are due to the conjugated $\pi$-linker, which can transfer electrons from the donor to the acceptor $[12,16]$. Researchers have continuously explored and improved the structure of these three parts to design a large number of D- $\pi$-A dye sensitizers. Eric Wei Guang diau, Chen Yu Yeh and coworkers designed highly efficient porphyrin dye sensitizer YD14 (as shown in Fig. 2) which belongs to D$\pi$-A structure [17].

The $\pi$-linker generally has good planar and conjugation properties, which is conducive to the electron transfer. And $\pi$-linker can improve the excitation efficiency and increase the lifetime of the excited electrons to a certain extent. The photoelectric properties of the $\pi$ linker can be improved by changing the length or dihedral angle of the $\pi$-linker, and then the optical properties of the dye sensitizer can be changed [18]. In 2008, Michael Grätzel, Shaik M. Zakeeruddin, Peng Wang and coworkers reported two new heteroleptic polypyridyl ruthenium complexes, coded $\mathrm{C} 101$ and $\mathrm{C} 102$ which are belong to D- $\pi$-A dye sensitizers (as shown in Fig. 2) [19]. The $\mathrm{C} 101$ sensitizer has already achieved a high efficiency of $11.0-11.3 \%$, along with acetonitrile-based electrolyte.

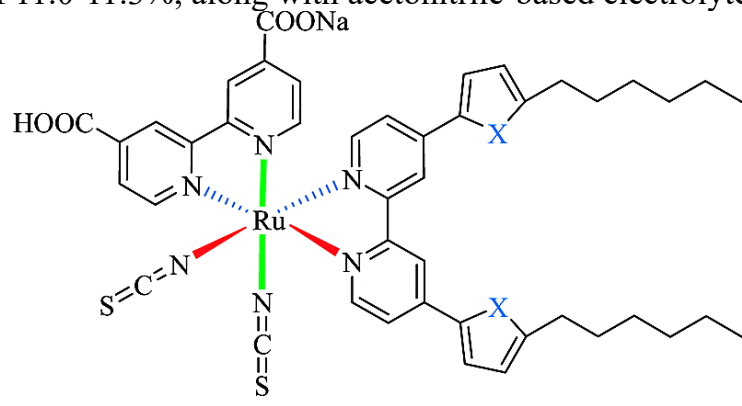

Fig. 2. The structures of $\mathrm{C} 101(\mathrm{X}=\mathrm{S})$ and $\mathrm{C} 102(\mathrm{X}=\mathrm{O})$.

It can be seen that $\pi$-linker plays an important role in improving the photovoltaic performance of dye, promoting the interaction between dye and $\mathrm{TiO}_{2}$ surface, and improving the photoelectric conversion efficiency of DSSCs. This result provides us with a new idea of designing new dye sensitizers by studying and improving $\pi$-linker structure.

\section{Composition classifications of dye sensitizers}

Dye sensitizers can be divided into the following two types, metal complex dye sensitizer and metal free organic dye sensitizer, according to whether they contain metal elements [20]. Metal complex dye sensitizers are mainly concentrated in ruthenium and osmium metal polypyridyl complexes, metal porphyrins, phthalocyanines and other substances. Metal free organic dye sensitizers include natural dyes and organic synthetic dyes [21]. 


\subsection{Metal complex sensitizer}

Since the report of high performance DSSCs containing ruthenium dye sensitizer by Grätzel research group in 1991, DSSCs have been widely studied because of their low cost, good flexibility and high efficiency, among which ruthenium dye sensitizer has also attracted great attention. In 1993, the group continued to study a series of sensitizers, among them, the red dye sensitizer which sensitized $\mathrm{TiO}_{2}$ nanocrystalline electrode and produced more than $80 \%$ incident photon-to-electron conversion efficiency (IPCE) in a wide wavelength range (480$600 \mathrm{~nm}), 17 \mathrm{~mA} / \mathrm{cm}^{2}$ short circuit current, $0.72 \mathrm{v}$ open circuit voltage and $10 \%$ total energy conversion density under simulated AM1.5 irradiation. In 2008, Peng Wang, Michael Grätzel and coworkers designed and synthesized a polypyridyl ruthenium complex C101 dye, which has made the photoelectric conversion efficiency of DSSCs as high as $11.3 \%$.

\subsection{Metal-free organic sensitizer}

With the development of research, it has been found that the molar absorption coeffcient of ruthenium-based sensitizer is relatively low, which lead to the loss of electrons at the interface of dye and semiconductor film, while the metal-free organic sensitizer has a large molar absorption coefficient [22]. In addition to the low molar absorption coeffcient, the metal elements (such as ruthenium) of metal complex dye sensitizers are relatively low content in nature, which greatly increases the manufacturing cost of this kind of dye sensitizer solar cells, which is not conducive to widely put into production and life. And ruthenium based dye sensitizers have great environmental hazards. Therefore, metal free organic dye sensitizers have attracted extensive attention due to their unique electrochemical advantages such as low toxicity and price, high molar extinction coefficient and environmental friendliness [23]. In recent years, the metalfree sensitizers, such as coumarin [24], porphyrin [25], indole dyes [26] and so on.

In 2003, Akrakawa team used cyanine dyes Cy0, Cy1 and partial cyanine dye SQ to co-sensitize $\mathrm{TiO}_{2}$ for the first time. It was found that different electrolytes had a great influence on the dye adsorption. When cholic acid was added to the acetonitrile electrolyte solution, the efficiency of the co-sensitized cell was improved, and the conversion efficiency could reach $3.1 \%$ under the condition of AM1.5 [27]. In the same year, Akrakawa team used coumarin dye as sensitizer of solar cells, and the photoelectric conversion efficiency reached $7.7 \%$ [28].

In 2009, Peng Wang developed the new DSSC with new high molar absorption coefficient organic dye $\mathrm{C} 217$ [29]. C217 dye uses ipophilic dihexyloxy-substituted triphenylamine as donor, hydrophilic cyanoacrylic acid as acceptor, and a binary spacer of orderly conjugated 3,4ethyldioxythiophene and thienothiophene as $\pi$-linker. When acetonitrile was used as electrolyte solvent, the photoelectric conversion efficiency of C217 DSSC reached $9.8 \%$.

Till 2019, Anders Hagfeldt, Licheng Sun, Xichuan Yang and coworkers designed two efficient metal-free organic dye sensitizers with one containing a flexible Ztype double bond and another a rigid single bond, coded as ZL001 and ZL003 respectively (as shown in Fig. 3) [30] The devices based on ZL003 exhibited the champion power conversion efficiency (PCE) of 13.6\%.

These conclusions show that metal-free organic sensitizers have great potential. Through our research and analysis, we believe that the performance of this type of dye sensitizer will achieve great achievements, and further improve the photoelectric conversion efficiency of DSSCs.

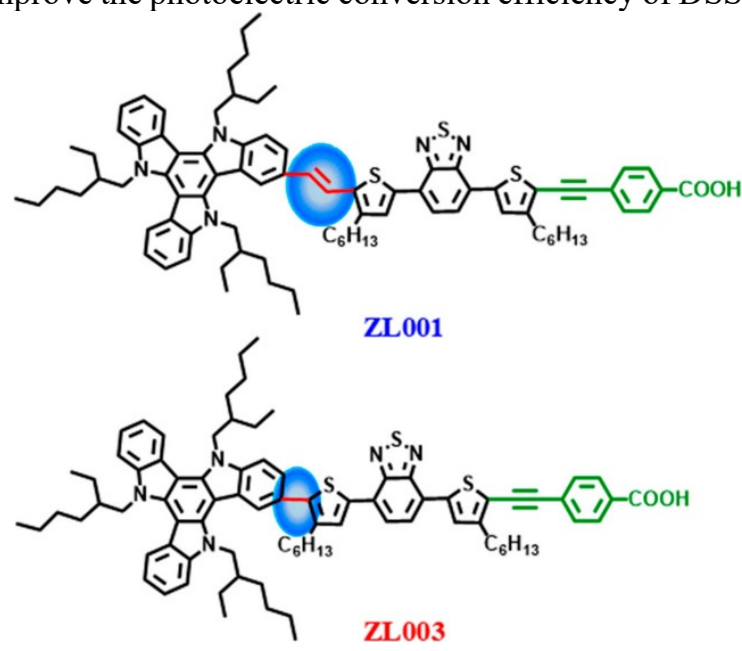

Fig. 3. The structures of ZL001 and ZL003.

\section{Application of theoretical chemical calculation in DSSCs}

Applying theoretical chemistry calculation to DSSCs research and development can not only explain and sublimate the experimental results, but also provide guidance and direction for the next experiment, improve the efficiency of scientific research and achieve the effect of double the effort. Density functional theory (DFT) has been widely accepted as an important theoretical calculation method suitable for the study of the structure and properties of tremendous systems.

For example, Michael Grätzel et al. modified the conjugated $\pi$-linker of two dye sensitizers by combining theoretical calculation and experiment, which further improved the photovoltaic performance of DSSCs [31]. In their research, DFT was used to optimize the structures of the two dye sensitizers, and the frontier orbital distribution and energy values were calculated. This result well supports the experimental data and explain the phenomenon that the two dyes have almost the same charge transfer rate which is found in the photoelectric character of the experiment. It is also fully proved that the analysis of dye sensitizer about HOMO and LUMO by theoretical calculation can help to judge the transfer trend of electrons in the sensitizer molecule. Hongxing Zhang and co-workers have studied a series of D - $\pi$ - A type metal free organic dye sensitizers with the same $\mathrm{D}$ and $\pi$ structure but different anchoring groups [32]. They fully demonstrated the photoelectric characteristics of the dye sensitizer itself and the electron transfer characteristics at the interface after combining with the semiconductor 
$\left(\mathrm{TiO}_{2}\right)_{16}$ cluster.

\section{Conclusions}

Dye sensitized solar cells (DSSCs), as a kind of universal, efficient and cheap solar cells have been widely favored by researchers since they were invented. Among the components of DSSCs, dye sensitizers play an important role. In order to improve the overall performance of the battery, researchers have been committed to developing more excellent dye sensitizers. In this paper, some new dye sensitizers which have been outstanding in recent years are summarized. The unique advantages and great potential of metal free pure organic D- $\pi$-A dyes and the important role of $\pi$-linker in the structure are emphatically affirmed. At the same time, the positive significance of theoretical chemical calculation in promoting the development of DSSCs is also illustrated. Therefore, we can use theoretical chemical calculation to reduce the waste of experimental resources and improve the efficiency, so as to provide reliable theoretical basis and guidance for experimental directional synthesis.

\section{References}

1. S. Chu, A. Majumdar. Nature. 488, 294 (2012).

2. A. Laurent, N. Espinosa. Energy \& Environmental Science. 8, 689 (2015).

3. T W. Hamann, R A. Jensen, A B. F. Martinson, H V. Ryswyk, J T. Hupp. Energy \& Environmental Science. 1, 66 (2008).

4. Michael Grätzel. Journal of The Electrochemical Society. 133, 331(1986).

5. M. Grätzel. Helvetica Chimica Acta. 67, 1012(1984).

6. M. Grätzelt, J. Desilvestro. Nature. 353, 737 (1991).

7. X. L. Wang, M. Wu, J. Ding, Z. S. Li, K. N. Sun. Journal of Power Sources. 246, 10 (2014).

8. H. W. Chen, C. Y. Hsu, J. G. Chen, K. M. Lee, C. C. Wang, K. C. Huang, K. C. Ho. Journal of Power Sources. 195, 6225 (2010).

9. J. M. Ji, H. Zhou, H. K. Kim. Journal of Materials Chemistry A. 6, 14518 (2018).

10. V. Sugathan, E. John, K. Sudhakar. Renewable and Sustainable Energy Reviews. 52, 54 (2015).

11. B. S. Chen, D. Y. Chen, C. L. Chen, C. W. Hsu, H. C. Hsu, K. L. Wu, S. H. Liu, P. T. Chou, Y. Chi. Journal of Materials Chemistry. 21, 1937 (2011).

12. Y. h. Tang, P. Shen, T. Ding, H. Huang, B. Zhao, S. Tan. European Polymer Journal. 46, 2033 (2010).

13. P. Singh, A. Baheti, K. R. Justin Thomas, C. P. Lee, K. C. Ho. Dyes and Pigments. 95,523 (2012).

14. W. d. Zeng, Y. m. Cao, Y. Bai, Y. h Wang, Y. s. Shi, M. Zhang, F. f. Wang, C. y. Pan, P. Wang. Chemistry of Materials. 22, 1915 (2010).

15. J. A. Mikroyannidis, G. Charalambidis, A. G. Coutsolelos, P. Balraju, G. D. Sharma. Journal of Power Sources. 196, 6622 (2011).
16. K. Srinivas, C. R. Kumar, M. A. Reddy, K. Bhanuprakash, V. J. Rao, L. Giribabu. Synthetic Metals. 161, 96 (2011).

17. S. L. Wu, H. P. Lu, H. T. Yu, S. H. Chuang, C. L. Chiu, C. W. Lee, E. W. G. Diau, C. Y. Yeh. Energy \& Environmental Science. 3, 949 (2010).

18. H. Zhou, P. c. Xue, Y. Zhang, X. Zhao, J. h. Jia, X. f. Zhang, X. 1. Liu, R. Lu. Tetrahedron. 67, 8477 (2011).

19. F. Gao, Y. Wang, D. Shi, J. Zhang, M. Wang, X. Jing, R. Humphry-Baker, P. Wang, S. M. Zakeeruddin, M. Gratzel. Journal of the American Chemical Society. 130, 10720 (2008).

20. J. Z. Zhang, J. Zhang, H. B. Li, Y. Wu, H. L. Xu, M. Zhang, Y. Geng, Z. M. Su. Journal of Power Sources. 267, 300 (2014).

21. H. Chang, Y. J. Lo. Solar Energy. 84, 1833 (2010).

22. G. D. Sharma, M. S. Roy, S. P. Singh. Journal of Materials Chemistry. 22, 18788 (2012).

23. R. Sirohi, D. H. Kim, S. C. Yu, S. H. Lee. Dyes and Pigments. 92, 1132 (2012).

24. K. Hara, K. Sayama, H. Arakawa, Y. Ohga, A. Shinpo, S. Suga. Chemical Communications. 569 (2001).

25. M. P. Balanay, C. V. P. Dipaling, S. H. Lee, D. H. Kim, K. H. Lee. Solar Energy Materials and Solar Cells. 91, 1775 (2007).

26. T. Horiuchi, H. Miura, K. Sumioka, S. Uchida. Journal of the American Chemical Society. 126, 12218 (2004).

27. K. Sayama, S. Tsukagoshi, T. Mori; K. Hara, Y. Ohga, A. Shinpou, Y. Abe, S. Suga, H. Arakawa. Solar Energy Materials and Solar Cells. 80, 47 (2003).

28. K. Hara, M. Kurashige, Y. Dan-oh, C. Kasada, A. Shinpo, S. Suga, K. Sayama, H. Arakawa. New Journal of Chemistry. 27, 783 (2003).

29. G. Zhang, H. Bala, Y. Cheng, D. Shi, X. Lv, Q. Yu, P. Wang. Chem Commun (Camb). 2009, 2198 (2009).

30. L. C. Zhang, X. C. Yang, W. H. Wang, G. G. Gurzadyan, J. J. Li, X. X. Li, J. C. An, Z. Yu, H. X. Wang, B. Cai, A. Hagfeldt, L. C. Sun. ACS Energy Letters. 4, 943 (2019).

31. S. Haid, M. Marszalek, A. Mishra, M. Wielopolski, J. Teuscher, J. E. Moser, R. Humphry-Baker, S. M. Zakeeruddin, M. Grätzel, P. Bäuerle. Advanced Functional Materials. 22, 1291 (2012).

32. T. F. Lu, W. Li, J. Chen, J. F. Tang, F. Q. Bai, H. X. Zhang. Electrochimica Acta. 283, 1798 (2018). 